\title{
Complex Inpatient Pain Management in a Critically Ill Lung Transplant Recipient: A Case Report
}

\author{
Tina Vajdi*, Paul Lee, Mary Alice Vijjeswarapu \\ Cedars-Sinai Medical Center, Department of Anesthesiology, Los Angeles, CA, USA \\ Email: *tina.vajdi@cshs.org
}

How to cite this paper: Vajdi, T., Lee, P., and Vijjeswarapu, M.A. (2019) Complex Inpatient Pain Management in a Critically Ill Lung Transplant Recipient: A Case Report. Case Reports in Clinical Medicine, 8, 157-163.

https://doi.org/10.4236/crcm.2019.86019

Received: April 5, 2019

Accepted: June 21, 2019

Published: June 24, 2019

Copyright () 2019 by author(s) and Scientific Research Publishing Inc. This work is licensed under the Creative Commons Attribution International License (CC BY 4.0). http://creativecommons.org/licenses/by/4.0/

\begin{abstract}
Thoracic surgery is known to cause severe pain that may not subside during the course of hospitalization. Early pain control is important in these patients because it improves ventilation and promotes mobility. Lung transplant recipients can experience post-operative complications that lead to extended hospital stays. This increases the time a patient is sedentary, which further deconditions patients; therefore, rehabilitation should be initiated early post-operatively. We present a unique case of a critically ill double lung transplant recipient whose rehabilitation post-operatively was hindered by severe vasopressor-induced ischemic pain. Due to debilitating pain, he was unable to regain mobility. His pain management course was challenging due to sensitivity to opioids, renal failure, and anticoagulation.
\end{abstract}

\section{Keywords}

Pain Management, Regional Anesthesia, Lung Transplant, Ischemic Pain

\section{Introduction}

Bilateral lung transplant recipients receive bilateral thoracosternotomy (clamshell) incisions that are associated with severe pain. Some patients report continuous pain months to years post-operatively that limits their mobility [1] [2]. Early pain control is important in these patients because it can improve ventilation and promotes mobility. Post-transplant, patients can have complications such as massive blood loss, infection, ventilator dependence, and organ rejection that lead to extended hospital stays. Prolonged hospitalization increases the time a patient is sedentary and further deconditions patients; therefore, rehabilitation should be initiated early post-operatively. Early physical therapy and mobility 
post-operatively can lead to improved quality of life and physical function [3] [4].

Pain can hinder a patient's mobility and recovery. In critically ill patients on vasopressors, ischemic skin necrosis can be an unfortunate complication. The poor tissue perfusion and bullous skin changes can cause unbearable, constant pain that is usually burning in nature. It can lead to devastating functional consequences, especially in the limbs. When surgical interventions such as debridement, amputation, and/or revascularization are not indicated, the main goal is treating the symptoms through pain management. Common ways to treat ischemic pain include targeting different pain receptors pharmacologically and with nerve blocks. We present a unique case of complex pain management in a critically ill lung transplant recipient who was sensitive to opioids, had renal failure, and was on anticoagulation. His significant pain limited his recovery post-operatively, and our inpatient pain management service played an integral role in his recovery.

\section{Case Overview}

The inpatient pain management service was consulted for a 64-year-old male with significant bilateral lower extremity ischemic pain. Our patient had a history of pulmonary fibrosis and had received a bilateral lung transplant a few weeks prior. His post-operative hospital course was complicated by mechanical ventilation and extracorporeal membrane oxygenation (ECMO) for worsening hypoxia, continuous renal replacement therapy (CRRT) and eventually hemodialysis (HD) for acute renal failure, and multiple vasopressors for hemodynamic instability. Unfortunately, he eventually developed ischemia of his bilateral toes and part of his feet (Figure $1 \&$ Figure 2). No surgical intervention for debridement or amputation was indicated.

Initially, the intensive care unit (ICU) team attempted managing his pain. However, there were significant limitations due to his critical status. He was unable to tolerate opioid medications because of significant ileus leading to sepsis, encephalopathy, and extreme somnolence. Our patient also had hemodynamic instability that precluded the use of medications such as dexmedetomidine and intravenous lidocaine. Podiatry had recommended topical therapies; however, due to the high risk of infection, the therapies were contraindicated. Prior to consultation with the inpatient pain management service, the patient's pain was being managed with a ketamine infusion and acetaminophen (Please refer to Figure 3 for ICU course by post-operative day). Given his waxing and waning mental status on this regimen, the patient was also being monitored in the ICU while on ketamine.

Given the significant limitations of the patient's recovery secondary to pain control, the ICU team consulted the inpatient pain management service to assist with optimizing care. Our in house pain management team assessed the patient and worked with the ICU to implement a thoughtful multimodal pain regimen 

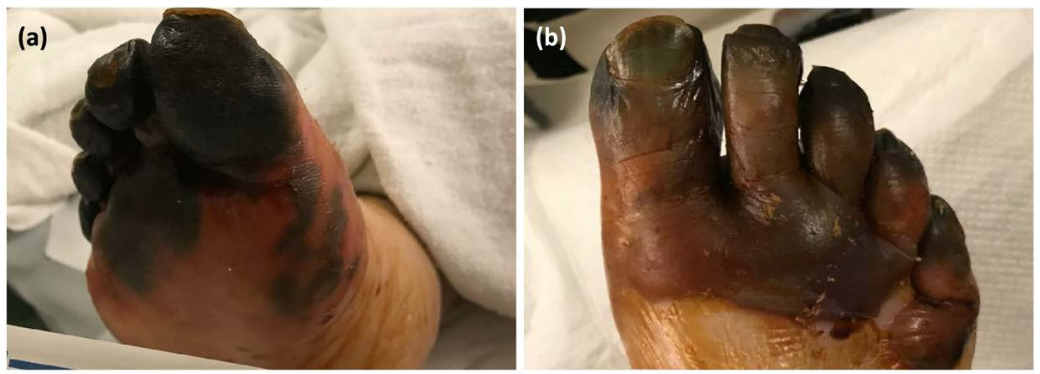

Figure 1. Severe cyanosis of the right forefoot spanning the digits to the metatarsal heads on the plantar (a) and dorsal (b) aspects of the foot. Fluid-filled bullae on the dorsum of the forefoot.
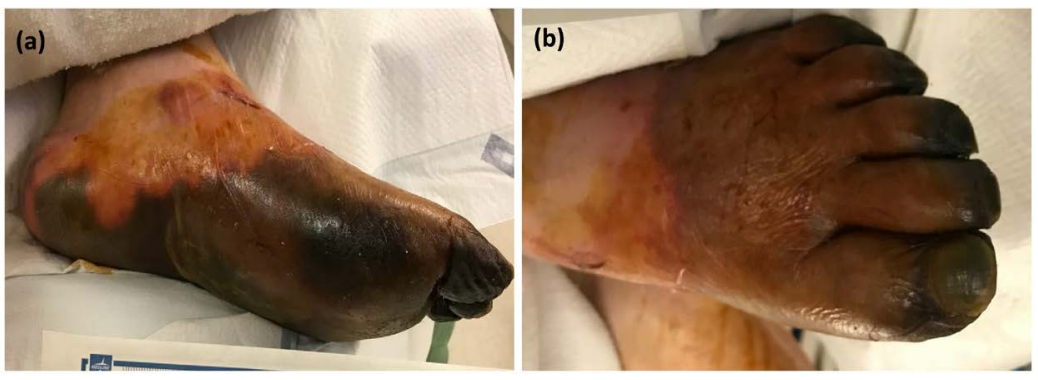

Figure 2. Severe cyanosis of the left forefoot and midfoot extending over the left ankle plantar (a) and dorsal (b) aspects of the foot. Fluid-filled bullae on distal, lateral foot.

\section{ICU Course By Post-Operative Day}

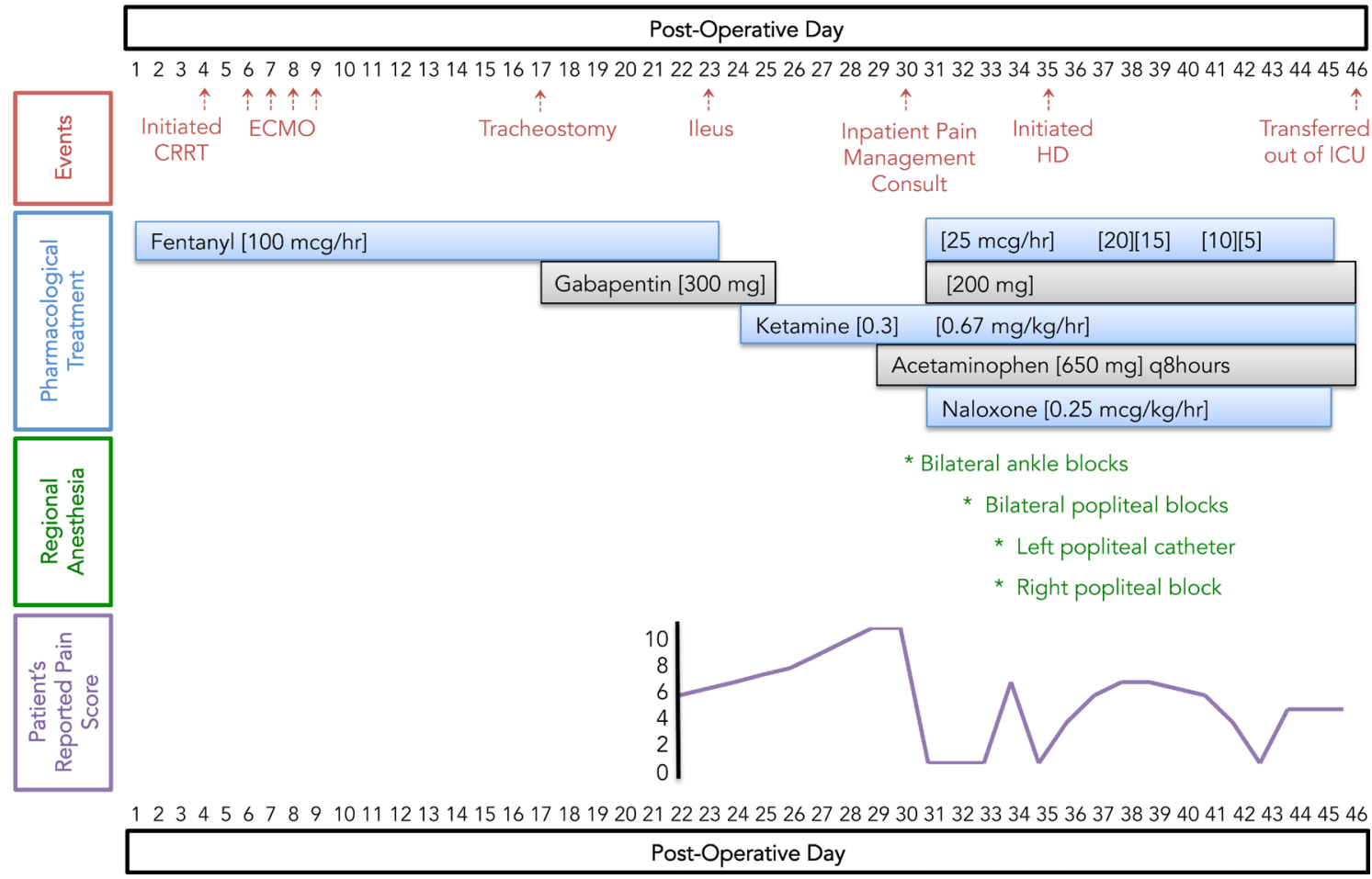

Figure 3. ICU Course by post-operative day. Events in the ICU are shown in red with arrows. Continuous pharmacological treatment is depicted with boxes spanning the post-operative days our patient received this pharmacological treatment. The brackets indicate the dosing administered. Regional anesthesia is shown in green with an asterisk $\left({ }^{*}\right)$, which indicates the post-operative day. Our patient's reported pain score is shown in purple on a scale of 0 through 10. Initially, he was sedated and/or too somnolent to participate in reporting his pain level. 
as described below.

For pain control, he was initially started on infusions of fentanyl at $25 \mathrm{mcg} / \mathrm{hr}$, ketamine at $0.67 \mathrm{mg} / \mathrm{kg} / \mathrm{min}$, and naloxone at $0.25 \mathrm{mcg} / \mathrm{kg} / \mathrm{hr}$ (for opioid-induced ileus per hospital protocol). Higher doses of fentanyl made him very somnolent and caused altered mental status. These pharmacologic treatments provided minimal analgesia for our patient. At the same time, he was on a heparin infusion due to multiple deep vein thrombosis (DVTs), which limited the procedural blocks we could offer. We considered neuraxial blockade, but given his need for a heparin infusion, we opted to not pursue this route. Bilateral ankle blocks with bupivacaine $0.25 \%$ were provided; however, they gave him only 5 hours of pain relief. Although short lived, this did provide the patient with a full night's sleep, and afterwards, he had a significant improvement in his mental status. Two days later, popliteal blocks were administered which temporarily reduced his pain (Please see Figure 3 for patient's reported pain score by post-operative day). The decision was made to temporarily hold his heparin infusion for 6 hours for bilateral popliteal nerve block catheter. The catheter could only be threaded on the left side, so he received $0.25 \%$ bupivacaine, which was started at $4 \mathrm{cc} /$ hour and weaned down over the course of five days. He received a single shot popliteal block on his right leg. These nerve blocks along with infusions of fentanyl, which was reduced to 15 $\mathrm{mcg} / \mathrm{hr}$, ketamine at $0.67 \mathrm{mg} / \mathrm{kg} / \mathrm{min}$, naloxone at $0.25 \mathrm{mcg} / \mathrm{kg} / \mathrm{hr}$, and gabapentin $200 \mathrm{mg}$ solution significantly reduced our patient's pain level. Due to his renal dysfunction, gabapentin dosing and administration was carefully coordinated with the nephrologist. Through multi-modal pain management, he became able to comfortably tolerate weight-bearing activities and actively participate with physical therapy. He was also safely transferred out of the ICU for further recovery.

\section{Discussion}

The therapeutic goal of treating ischemic pain is to improve quality of life and functional status. In our patient's case, vasopressor-induced ischemia of the bilateral toes and feet caused relentless pain that he reported as significantly worse than the pain from the thoracic clamshell incisions across his chest for his bilateral lung transplant. In fact, once his organs began to recover, it was his challenging pain control that greatly hindered the recovery of an otherwise successful lung transplant surgery. It prevented him from working with physical therapy, leading to further deconditioning and poor quality of life.

For patients with inoperable ischemia, the initial step in treating ischemic pain is through pharmacologic means. Opioids are used to treat severe ischemic pain. Synthetic, short-acting opioids such as fentanyl and alfentanil can be safely administered to patients with kidney failure [5]. In order to prevent opioid side effects including constipation, delayed gastric emptying, and ileus, low doses of naloxone can be used simultaneously with the administration of opioids without affecting analgesia [6] [7].

Ischemic pain also has a component of neuropathic pain that can be treated with medications such as ketamine and gabapentin. Even a 4-hour infusion of 
ketamine can give patients significant ischemic pain relief for a few days following the infusion [8]. In another study, patients who received a median daily dose of $1271 \mathrm{mg}$ of gabapentin in addition to high dose opioids reported significant reductions in their pain scores [9]. Unfortunately, patients with renal failure are unable to take gabapentin as it is eliminated by renal excretion. The refractory nature of the ischemic pain coupled with our patient's acute kidney failure made it very difficult to treat the pain pharmacologically. We started him on a fentanyl infusion and soon after a ketamine infusion in hopes that the $\mu$-opioid agonist and NMDA antagonist properties would synergistically reduce his pain level. Eventually, we were able to start him on a low dose of gabapentin after his kidney function improved. Yet, he continued to have intolerable pain.

Interventional treatments should be considered when patients have ischemic pain that is refractory to pharmacologic management. Surgical or chemical sympathectomy can help reduce pain via vasodilation and subsequent improvement of blood flow to areas of ischemia to promote healing. Sympathectomy can also act on nociceptive fibers to block sympathetic-nociceptive coupling to reduce pain [10]. For upper extremities, a stellate ganglion block can be considered for sympathectomy. This block can be effectively used to treat pain caused by vascular insufficiency for patients with atherosclerosis, Reynaud's disease, and thromboembolism [11] [12]. For lower extremities, epidural or spinal anesthesia can cause a chemical sympathectomy. A recent study has found chemical sympathectomy to be as effective as surgical lumbar sympathectomy in controlling ischemic pain [13]. Unfortunately, we were unable to consider chemical sympathectomy such as an epidural for this patient because he was receiving therapeutic levels of heparin for known DVTs. The risk of hematoma in the spinal cord outweighed the benefits of potential ischemic pain relief; therefore, we considered other blocks instead.

Continuous peripheral nerve blocks can also be efficacious in treating ischemic pain [14]. A lumbar plexus block can be used as an alternative to chemical sympathectomy for the lower extremities. When compared to epidurals, a lumbar plexus block is a unilateral block that provides similar analgesia with fewer adverse effects such as hypotension, urinary retention, and nausea [15]. Although it is safer to perform a lumbar plexus block than an epidural or spinal block for patients that are taking anticoagulants, there is still a significant risk of bleeding that can go unnoticed given that this block is deep. The German and Austrian Societies of Anesthesiology recommend the same guidelines be used for deep plexus blocks and neuraxial blocks when patients are on anticoagulants [16]. Although we considered bilateral lumbar plexus blocks for our patient's ischemic pain, we ultimately decided that superficial blocks of the ankle and popliteal region would be safer initial therapy before considering deeper plexus blocks due to the anticoagulation he was receiving.

\section{Conclusion}

Thoughtful multi-modal pain management played a pivotal role in our patient's 
recovery after receiving a lung transplant. Despite the limitations due to opioid sensitivity, kidney failure, and anticoagulation, we were able to effectively control his pain through our inpatient pain management service. Once his pain was well controlled, he was downgraded from the ICU, able to ambulate with assistance, transitioned to oral pain medications, and reported improved quality of life. A few weeks later, he was discharged to a rehabilitation center.

\section{Conflicts of Interest}

The authors declare no conflicts of interest regarding the publication of this paper.

\section{References}

[1] Ochroch, E.A., Gottschalk, A., Augostides, J., Carson, K.A., Kent, L., Malayaman, N., Kaiser, L.R., Aukburg, S.J. (2002) Long-Term Pain and Activity during Recovery from Major Thoracotomy Using Thoracic Epidural Analgesia. Anesthesiology, 97, 1234-1244. https://doi.org/10.1097/00000542-200211000-00029

[2] Allan Gottschalk, Steven Cohen, Stephen Yang and Ochroch, E.A. (2006) Preventing and Treating Pain after Thoracic Surgery. Anesthesiology, 104, 594-600. https://doi.org/10.1097/00000542-200603000-00027

[3] Truong, A.D., Fan, E., Brower, R.G. and Needham, D.M. (2009) Bench-to-Bedside Review: Mobilizing Patients in the Intensive Care Unit-From Pathophysiology to Clinical Trials. Critical Care, 13, 216. https://doi.org/10.1186/cc7885

[4] Kayambu, G., Boots, R. and Paratz, J. (2013) Physical Therapy for the Critically Ill in the ICU: A Systematic Review and Meta-Analysis. Critical Care Medicine, 41, 1543-1554. https://doi.org/10.1097/CCM.0b013e31827ca637

[5] Tawfic, Q. and Bellingham, G. (2015) Postoperative Pain Management in Patients with Chronic Kidney Disease. Journal of Anaesthesiology Clinical Pharmacology, 31, 6-13. https://doi.org/10.4103/0970-9185.150518

[6] Gan, T., Ginsberg, B., Glass, P., Fortney, J., Jhaveri, R. and Perno, R. (1997) Opioid-Sparing Effects of a Low-Dose Infusion of Naloxone in Patient-Administered Morphine Sulfate. Anesthesiology, 87, 1075-1081.

https://doi.org/10.1097/00000542-199711000-00011

[7] Xiao, Y., Wu, L., Zhou, Q., Xiong, W., Duan, X. and Huang, X. (2015) A Randomized Clinical Trial of the Effects of Ultra-Low-Dose Naloxone Infusion on Postoperative Opioid Requirements and Recovery. Acta Anaesthesiologica Scandina, 59, 1194-1203. https://doi.org/10.1111/aas.12560

[8] Mitchell, A.C. and Fallon, M.T. (2002) A Single Infusion of Intravenous Ketamine Improves Pain Relief in Patients with Critical Limb Ischaemia: Results of a Double Blind Randomised Controlled Trial. Pain, 97, 275-281. https://doi.org/10.1016/S0304-3959(02)00033-7

[9] Morris-Stiff, G. and Lewis, M. (2010) Gabapentin (Neurontin) Improves Pain Scores of Patients with Critical Limb Ischaemia: An Observational Study. International Journal of Surgery, 8, 212-215. https://doi.org/10.1016/j.ijsu.2009.12.009

[10] Nesargikar, P., Ajit, M., Eyers, P., Nichols, B. and Chester, J. (2009) Lumbar Chemical Sympathectomy in Peripheral Vascular Disease: Does It Still Have a Role? International Journal of Surgery, 7, 145-149. https://doi.org/10.1016/j.ijsu.2009.01.004

[11] Kulkarni, K.R., Kadam, A.I. and Namazi, I.J. (2010) Efficacy of Stellate Ganglion 
Block with an Adjuvant Ketamine for Peripheral Vascular Disease of the Upper Limbs. Indian Journal of Anaesthesia, 54, 546-551.

https://doi.org/10.4103/0019-5049.72645

[12] Zenz, M., Tryba, M. and Horch, C. (1986) Sympathetic Block after Plexus Anesthesia. Comparison with Stellate Ganglion Block. Regional Anaesthesie, 9, 84-87.

[13] Piatko, V. and Sukhotin, S. (2004) Chemical Lumbar Sympathectomy in Patients with Chronic Ischemia of the Lower Extremities. Anesteziologiia I Reanimatologiia, 31-33.

[14] Hashimoto, A., Ito, H., Sato, Y. and Fujiwara, Y. (2013) The Efficacy and Safety of Continuous Popliteal Sciatic Nerve Block for the Relief of Pain Associated with Critical Limb Ischemia: A Retrospective Study. Open Journal of Anesthesiology, 3, 433-437. https://doi.org/10.4236/ojanes.2013.310090

[15] Turker, G., Uckunkaya, N., Yavascaoglu, B., Yilmazlar, A. and Ozcelik, S. (2003) Comparison of the Catheter-Technique Psoas Compartment Block and the Epidural Block for Analgesia in Partial Hip Replacement Surgery. Acta Anaesthesiologica Scandinavica, 47, 30-36. https://doi.org/10.1034/j.1399-6576.2003.470106.x

[16] Martins, L.E., Ferraro, L.H., Takeda, A., Munechika, M. and Tardelli, M.A. (2017) Ultrasound-Guided Peripheral Nerve Blocks in Anticoagulated Patients-Case Series. Brazilian Journal of Anesthesiology, 67, 100-106.

https://doi.org/10.1016/j.bjan.2016.02.005 